Supporting Information for

\title{
Determination of Global Structure from Distance and Orientation Constraints in Biological Solids using Solid-State NMR Spectroscopy
}

\author{
Loren B. Andreas, ${ }^{1}$ Anil K. Mehta, ${ }^{2 *}$ Manish A. Mehta ${ }^{1 *}$ \\ ${ }^{1}$ Department of Chemistry and Biochemistry, Oberlin College, Oberlin, OH 44074 \\ ${ }^{2}$ Department of Chemistry, Emory University, Atlanta, GA 30322 \\ E-mail: anil.mehta@emory.edu; manish.mehta@oberlin.edu
}

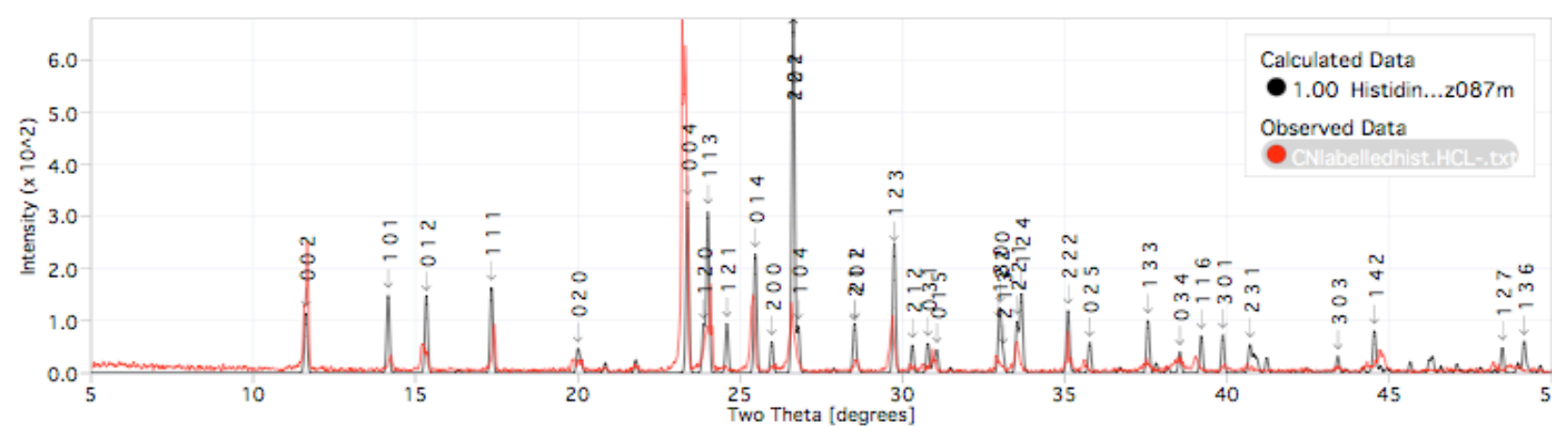

Figure S1. Overlay of calculated (black) and observed (red) X-Ray powder patterns of LHistidine $\cdot \mathrm{HCl} \cdot \mathrm{H}_{2} \mathrm{O}$. Peaks at $38^{\circ}$ and $44.5^{\circ}$ are background signal from the aluminum sample holder. The collected powder pattern was compared with that predicted from the experimentally determined crystal structure in order to confirm that all NMR samples had the same polymorph. 

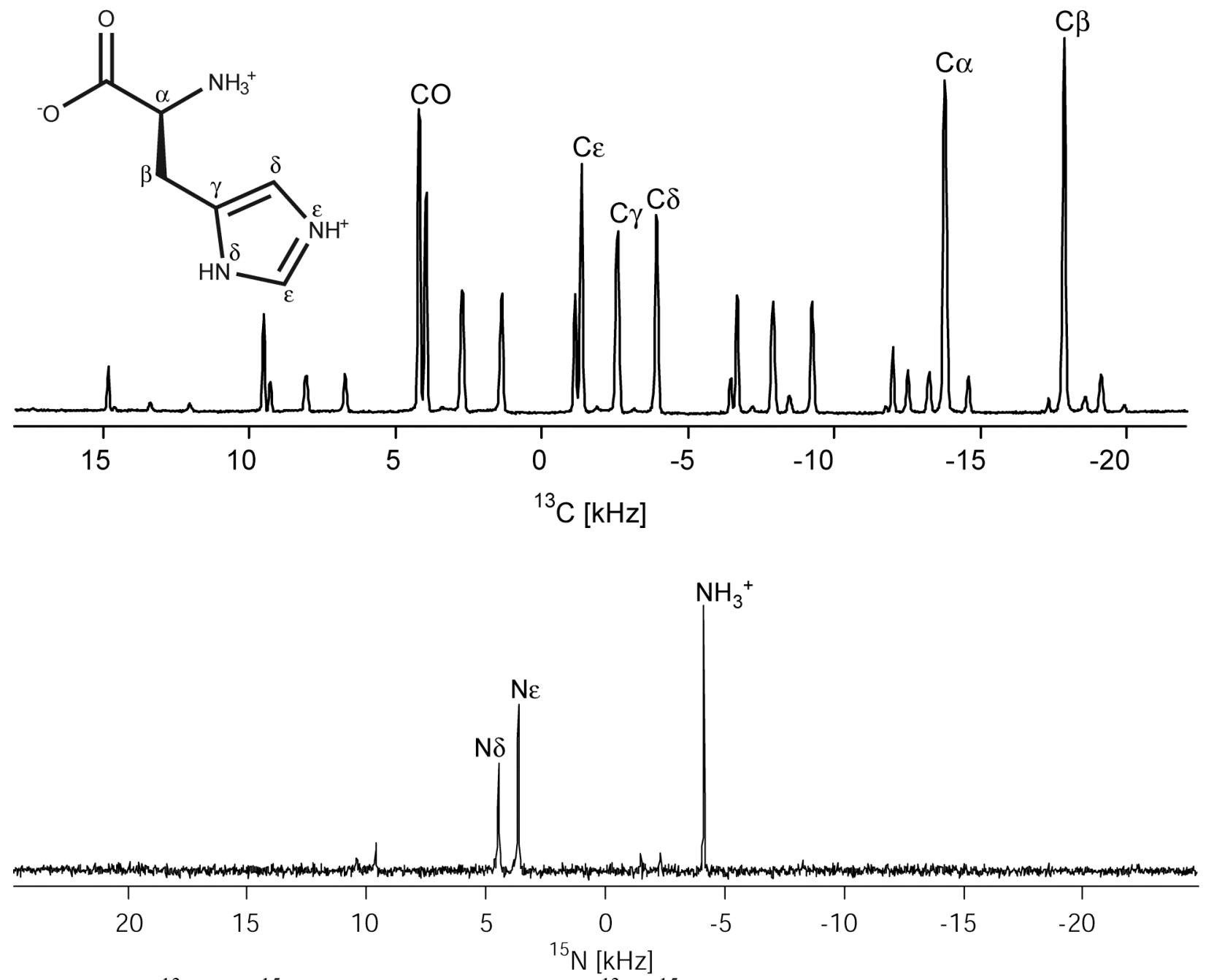

Figure S2. ${ }^{13} \mathrm{C}$ and ${ }^{15} \mathrm{~N}$ CP-MAS spectra of $\mathrm{U}-\left[{ }^{13} \mathrm{C}_{6},{ }^{15} \mathrm{~N}_{3}\right]$-L-Histidine $\cdot \mathrm{HCl} \cdot \mathrm{H}_{2} \mathrm{O}$, diluted 1:9 with natural abundance histidine, with MAS $v_{\mathrm{r}}=5319 \mathrm{~Hz}$ and $100 \mathrm{kHz}$ SPINAL-64 ${ }^{1} \mathrm{H}$ decoupling. 


\section{SPECTRAL ASSIGNMENT}

We used a $2 \mathrm{D}^{13} \mathrm{C}-{ }^{13} \mathrm{C}$ homonuclear correlation experiment to assign the ${ }^{13} \mathrm{C}$ resonances. Many pulse sequences can be used to produce homonuclear double-quantum coherences, such as DRAWS ${ }^{1}, \mathrm{C}^{2}$, POST-C $7^{3}, \mathrm{CMR}-7^{4}, \mathrm{SPC}-5^{5}$. In the SPC-5 pulse sequence, $5{ }^{13} \mathrm{C}$ pulses of $2 \pi$ nutation are applied per rotor period. ${ }^{5}$ Thus the ${ }^{13} \mathrm{C}$ nutation frequency must be 5 times the rotor frequency. At high magnetic field strengths, it is desirable to spin the sample faster in order to reduce the number and intensities of spinning sidebands. This means that the SPC-5 pulse sequence should perform better than DRAWS $(8.52 \pi$ pulses per rotor period) and C7 (7 $2 \pi$ pulses per rotor period). The SPC- 5 sequence requires only $52 \pi$ pulses per rotor period, allowing lower carbon power or faster spinning than other related sequences.

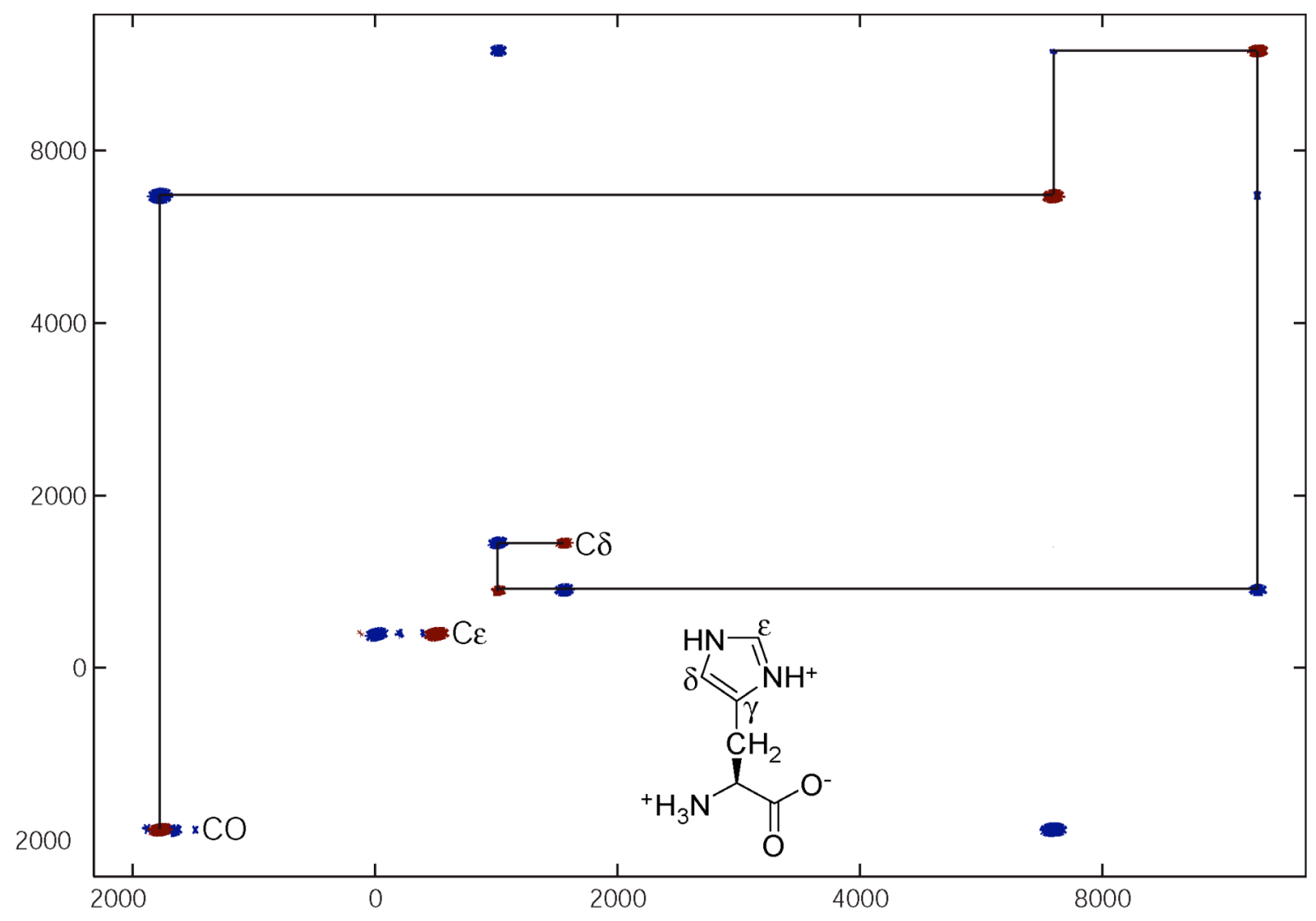

Figure S3. SPC-5 ${ }^{13} \mathrm{C}_{-13}{ }^{13} \mathrm{C}$ correlation spectrum of $\mathrm{U}-\left[{ }^{13} \mathrm{C}_{6}{ }^{15} \mathrm{~N}_{3}\right]-\mathrm{L}$-Histidine $\cdot \mathrm{HCl} \cdot \mathrm{H}_{2} \mathrm{O}$ with a 1 millisecond SPC-5 mixing time at $v_{\mathrm{r}}=8 \mathrm{kHz}$ MAS demonstrates the process of spectral assignment. Isotropic peaks appear along the diagonal and are colored red to represent positive amplitude. For the short mixing time used, only one-bond ${ }^{13} \mathrm{C}-{ }^{13} \mathrm{C}$ correlation peaks appear in the spectrum. These peaks are colored blue to represent negative amplitude. Each peak correlates two diagonal peaks, indicating connectivity. There is a spectral artifact where the center frequency intersects the shoulder of the epsilon carbon peak. 


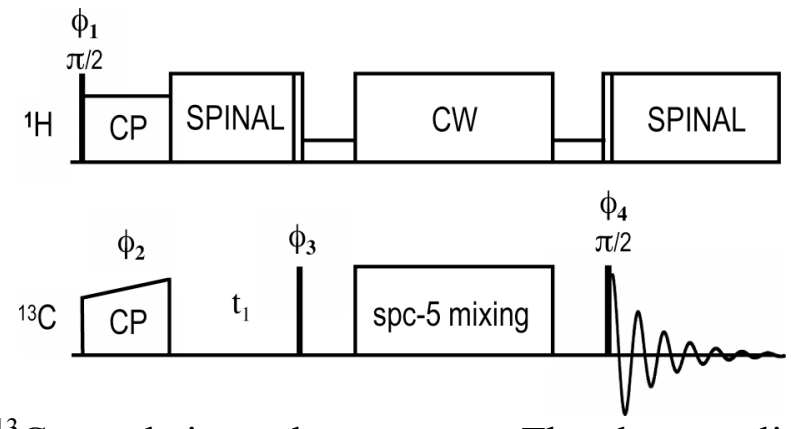

Figure S4. SPC- $5{ }^{13} \mathrm{C}-{ }^{13} \mathrm{C}$ correlation pulse sequence. The phase cycling is, $\phi_{1}=0000000022222222, \phi_{2}=02, \phi_{3}=1, \phi_{4}=00112233, \phi_{\text {rec }}=0213203120310213$. The phases within the SPC-5 mixing block were held constant from scan to scan. For details of the SPC-5 mixing block, see reference. ${ }^{5}$ 


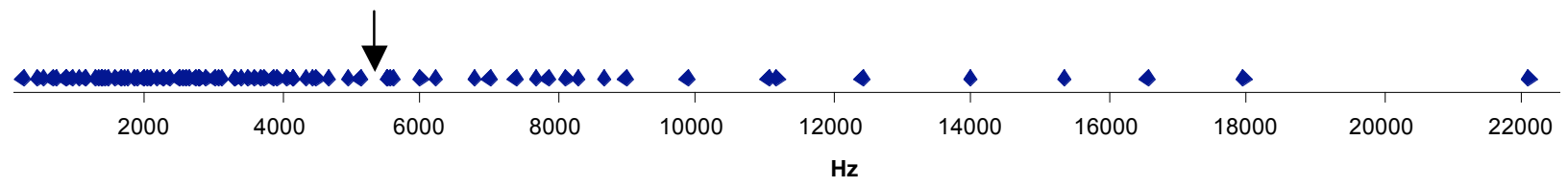

Figure S5. The rotational resonance conditions $\left(\mathrm{R}^{2}\right)$ between all homonuclear and heteronuclear spin pairs under $5 \AA$. Each blue point indicates the center of an $\mathrm{R}^{2}$ condition. The conditions were calculated using coordinates from the crystal structure and isotropic ${ }^{13} \mathrm{C}$ chemical shifts from Figure 2. The MAS speed used in the DANTE-REDOR experiments, 5319 $\mathrm{Hz}$, is indicated by the arrow. 


\section{CONFORMATIONAL SEARCHES}

The panels below show the resulting families of structures using different combinations of the experimental determined distance and angle restraints (main text Table 1). The carboxyl group and the $\mathrm{C}^{\prime}$ PAS were not allowed to move during the conformational search and therefore are identical for all structures. Points in white near the $\mathrm{C}^{\prime}$ (carboxyl) carbon indicate the principal axes of that carbon's CSA tensor frame of reference. The crystal structure is overlaid in red.
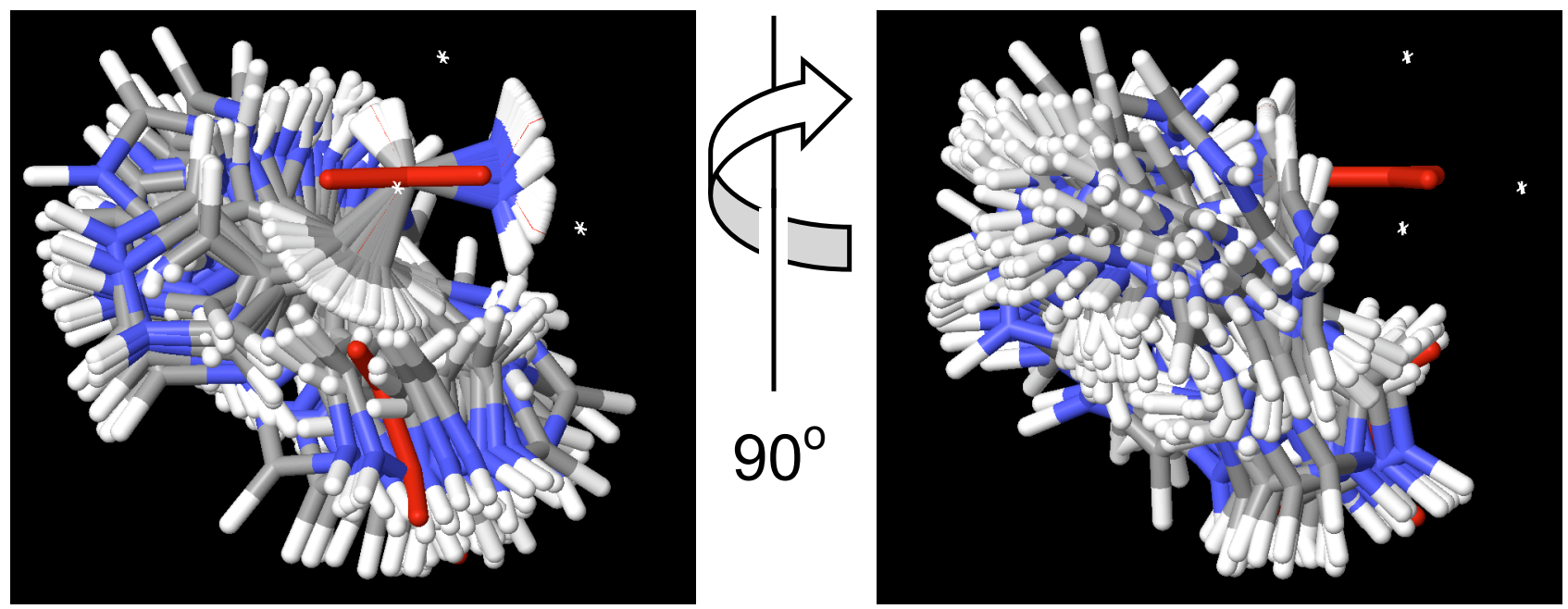

Figure S6. Restraint: ${ }^{13} \mathrm{C}^{\prime}-{ }^{15} \mathrm{~N}_{\delta}$ distance.
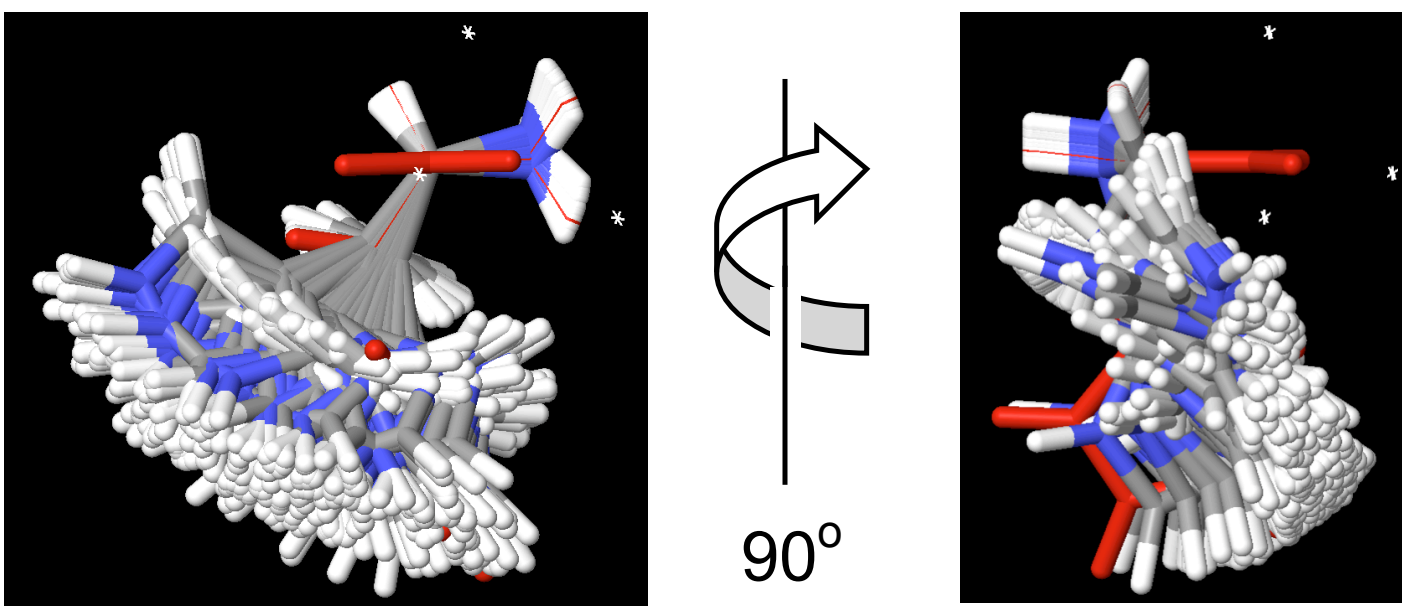

Figure S7. Restraint: ${ }^{13} \mathrm{C}^{\prime}-{ }^{15} \mathrm{~N}_{\varepsilon}$ distance. 

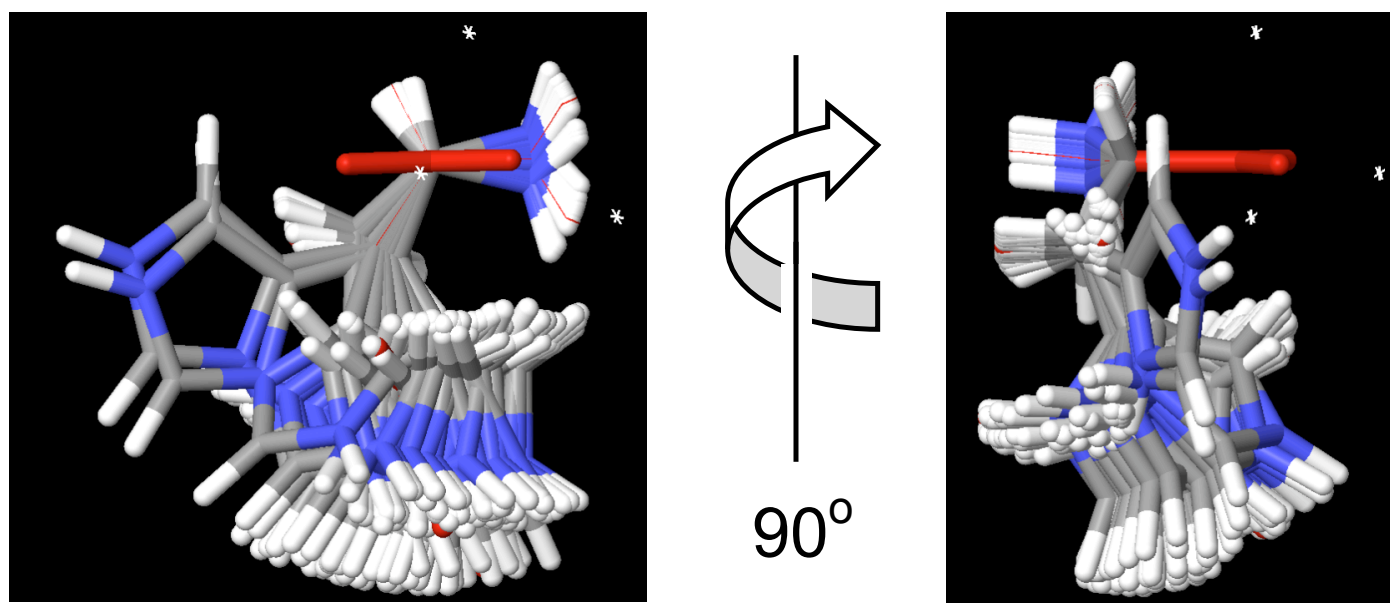

Figure S8. Restraints: ${ }^{13} \mathrm{C}^{\prime}-{ }^{15} \mathrm{~N}_{\delta}$ and ${ }^{13} \mathrm{C}^{\prime}-{ }^{15} \mathrm{~N}_{\varepsilon}$ distances.
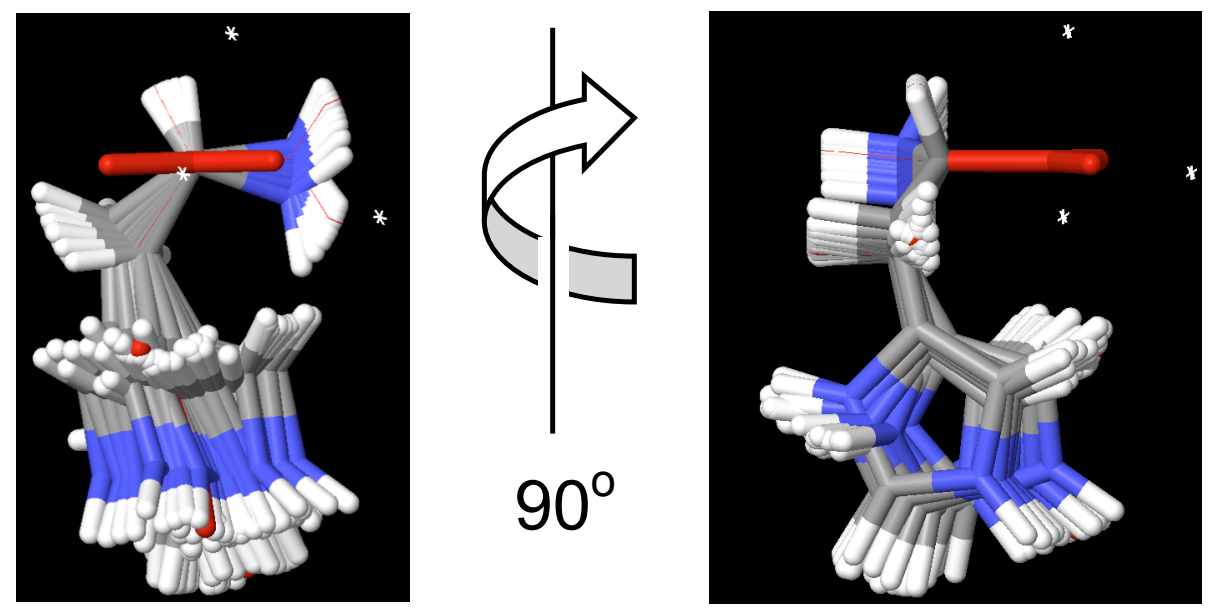

Figure 59. Restraints: ${ }^{13} \mathrm{C}^{\prime}-{ }^{15} \mathrm{~N}_{\delta}$ distances and angles.
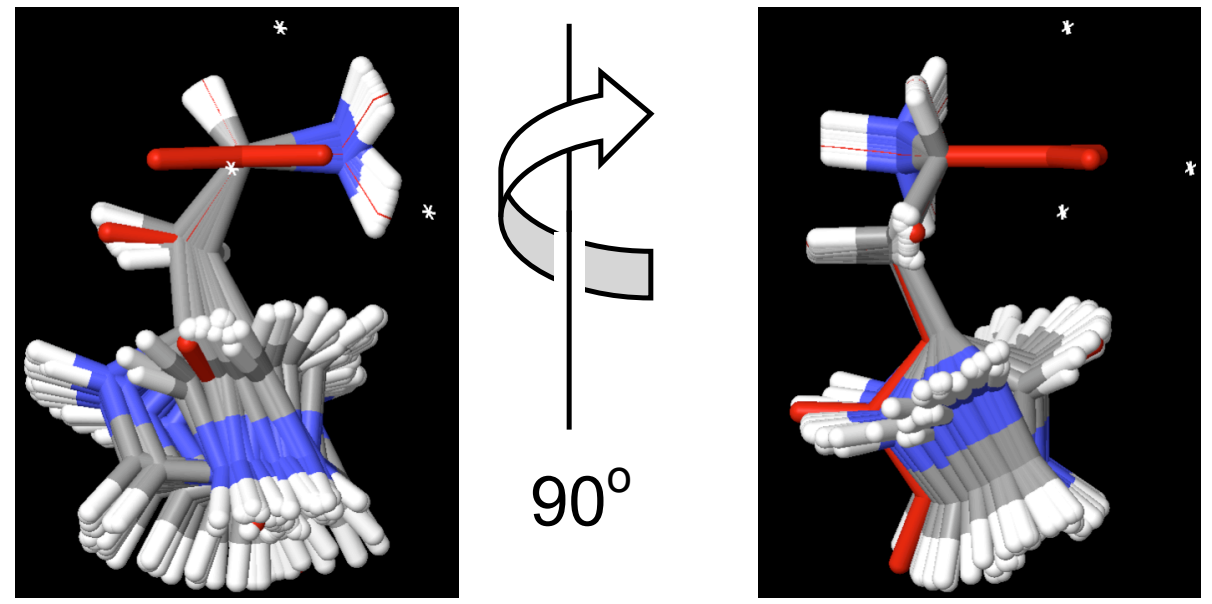

Figure S10. Restraints: ${ }^{13} \mathrm{C}^{\prime}-{ }^{15} \mathrm{~N}_{\varepsilon}$ distances and angles. 

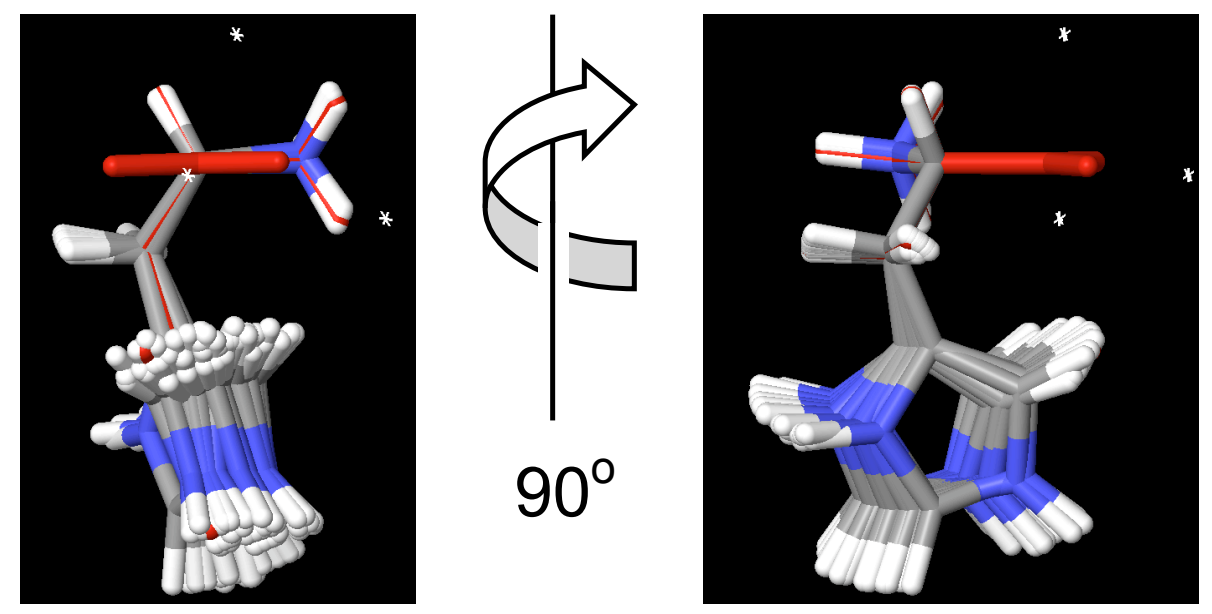

Figure S11. Restraints: ${ }^{13} \mathrm{C}^{\prime}-{ }^{15} \mathrm{~N}_{\delta}$ and ${ }^{13} \mathrm{C}^{\prime}-{ }^{15} \mathrm{~N}_{\varepsilon}$ angles.
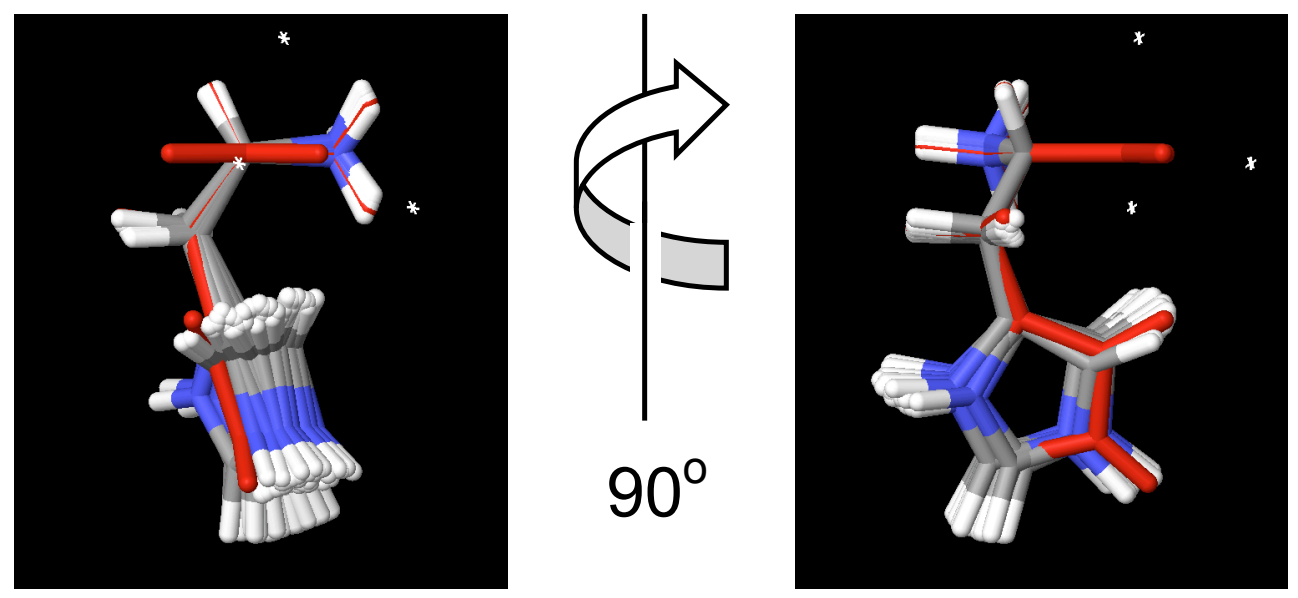

Figure S12. Restraints: ${ }^{13} \mathrm{C}^{\prime}-{ }^{15} \mathrm{~N}_{\delta}$ and ${ }^{13} \mathrm{C}^{\prime}-{ }^{15} \mathrm{~N}_{\varepsilon}$ distances and angles.

\begin{tabular}{|l|c|c|}
\hline \multicolumn{1}{|c|}{ Restraints } & $\begin{array}{c}\text { RMSD }(\AA) \\
\text { all C \& N } \\
\text { except } \mathrm{C}^{\prime}\end{array}$ & $\begin{array}{c}\text { RMSD }(\AA) \\
\text { imidazole } \\
\mathrm{C} \text { and } \mathrm{N}\end{array}$ \\
\hline${ }^{13} \mathrm{C}^{\prime}-{ }^{15} \mathrm{~N}_{\delta}$ distance & 3.05 & 5.08 \\
\hline${ }^{13} \mathrm{C}^{\prime}-{ }^{15} \mathrm{~N}_{\varepsilon}$ distance & 1.86 & 2.35 \\
\hline${ }^{13} \mathrm{C}^{\prime}-{ }^{15} \mathrm{~N}_{\delta}$ and ${ }^{13} \mathrm{C}^{\prime}-{ }^{15} \mathrm{~N}_{\varepsilon}$ distances & 0.97 & 1.75 \\
\hline${ }^{13} \mathrm{C}^{\prime}-{ }^{15} \mathrm{~N}_{\delta}$ distances and angles & 0.50 & 0.89 \\
\hline${ }^{13} \mathrm{C}^{\prime}-{ }^{15} \mathrm{~N}_{\varepsilon}$ distances and angles & 0.85 & 1.63 \\
\hline${ }^{13} \mathrm{C}^{\prime}-{ }^{15} \mathrm{~N}_{\delta}$ and ${ }^{13} \mathrm{C}^{\prime}-{ }^{15} \mathrm{~N}_{\varepsilon}$ angles & 0.42 & 0.75 \\
\hline${ }^{13} \mathrm{C}^{\prime}-{ }^{15} \mathrm{~N}_{\delta}$ and ${ }^{13} \mathrm{C}^{\prime}-{ }^{15} \mathrm{~N}_{\varepsilon}$ distances and angles & 0.32 & 0.59 \\
\hline
\end{tabular}

Table S1. Average RMSD for each family of structures with respect to the x-ray structure shown in Figures S6 to S12. RMSD includes only carbon and nitrogen atoms (excluding backbone $\left.\mathrm{C}^{\prime}\right)$ or imidazole carbon and nitrogen atoms $(\mathrm{C} \gamma, \mathrm{C} \delta, \mathrm{N} \delta, \mathrm{C} \varepsilon$ and $\mathrm{N \varepsilon})$. 


\section{REFERENCES}

(1) Mehta, M. A.; Gregory, D. M.; Kiihne, S.; Mitchell, D. J.; Hatcher, M. E.; Shiels, J. C.; Drobny, G. P. Solid State Nucl. Mag. Res. 1996, 7, 211-228.

(2) Lee, Y. K.; Kurur, N. D.; Helmle, M.; Johannessen, O. G.; Nielsen, N. C.; Levitt, M. H. Chem. Phys. Lett. 1995, 242, 304-309.

(3) Hohwy, M.; Jakobsen, H. J.; Eden, M.; Levitt, M. H.; Nielsen, N. C. J. Chem. Phys. 1998, 108, 2686-2694.

(4) Rienstra, C. M.; Hatcher, M. E.; Mueller, L. J.; Sun, B. Q.; Fesik, S. W.; Griffin, R. G. J. Am. Chem. Soc. 1998, 120, 10602-10612.

(5) Hohwy, M.; Rienstra, C. M.; Jaroniec, C. P.; Griffin, R. G. J. Chem. Phys. 1999, 110, 7983-7992. 


\section{SINGLE-CRYSTAL X-RAY DIFFRACTION INFORMATION}

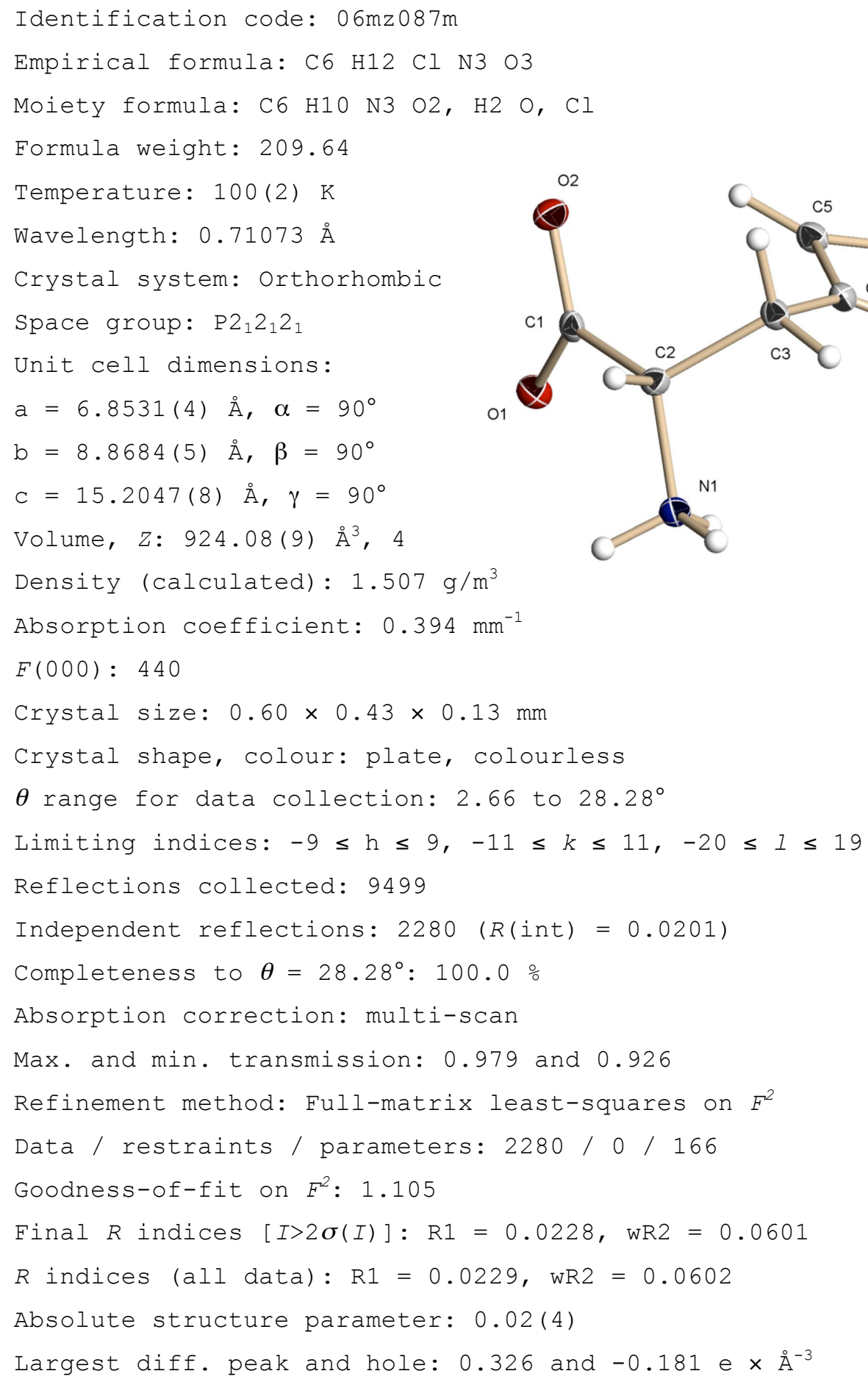


Refinement of $F^{2}$ against ALL reflections. The weighted R-factor wR and goodness of fit are based on $F^{2}$, conventional R-factors $\mathrm{R}$ are based on $F$, with $F$ set to zero for negative $F^{2}$. The threshold expression of $F^{2}>2 \sigma\left(F^{2}\right)$ is used only for calculating R-factors

Treatment of hydrogen atoms:

All hydrogen atoms were located in the difference density Fourier map and were isotropically refined.

Table 2. Atomic coordinates $\left[\times 10^{4}\right]$ and equivalent isotropic displacement parameters $\left[\AA^{2} \times 10^{3}\right]$ for $06 \mathrm{mz} 087 \mathrm{~m}$. U(eq) is defined as one third of the trace of the orthogonalized $U_{i j}$ tensor.

\begin{tabular}{|c|c|c|c|c|}
\hline & $\mathrm{x}$ & $\mathrm{Y}$ & $\mathrm{z}$ & $\mathrm{U}(\mathrm{eq})$ \\
\hline C (1) & $-2614(2)$ & $5575(1)$ & $8852(1)$ & $12(1)$ \\
\hline$C(2)$ & $-791(2)$ & $6358(1)$ & $8479(1)$ & $11(1)$ \\
\hline$C(3)$ & $558(2)$ & $5278(1)$ & $7979(1)$ & $12(1)$ \\
\hline$C(4)$ & $1129(2)$ & $3892(1)$ & $8483(1)$ & $12(1)$ \\
\hline$C(5)$ & $29(2)$ & 2751 (1) & $8823(1)$ & $13(1)$ \\
\hline$C(6)$ & $3121(2)$ & $2237(1)$ & $9099(1)$ & $15(1)$ \\
\hline Cl (1) & $2853(1)$ & 4795 (1) & $5731(1)$ & $14(1)$ \\
\hline $\mathrm{N}(1)$ & $274(2)$ & $7149(1)$ & $9191(1)$ & $12(1)$ \\
\hline $\mathrm{N}(2)$ & $3055(2)$ & $3538(1)$ & $8661(1)$ & $13(1)$ \\
\hline $\mathrm{N}(3)$ & $1308(2)$ & $1744(1)$ & $9207(1)$ & $15(1)$ \\
\hline$O(1)$ & $-2953(1)$ & $5679(1)$ & 9651 (1) & $15(1)$ \\
\hline$O(2)$ & $-3605(1)$ & $4860(1)$ & $8285(1)$ & $17(1)$ \\
\hline$O(3)$ & $6619(2)$ & $3468(1)$ & $6677(1)$ & $23(1)$ \\
\hline
\end{tabular}

All esds (except the esd in the dihedral angle between two l.s. planes) are estimated using the full covariance matrix. The cell esds are taken into account individually in the estimation of esds in distances, angles and torsion angles; correlations between esds in cell parameters are only used when they are defined by crystal symmetry. An approximate (isotropic) treatment of cell esds is used for estimating esds involving l.s. planes. 


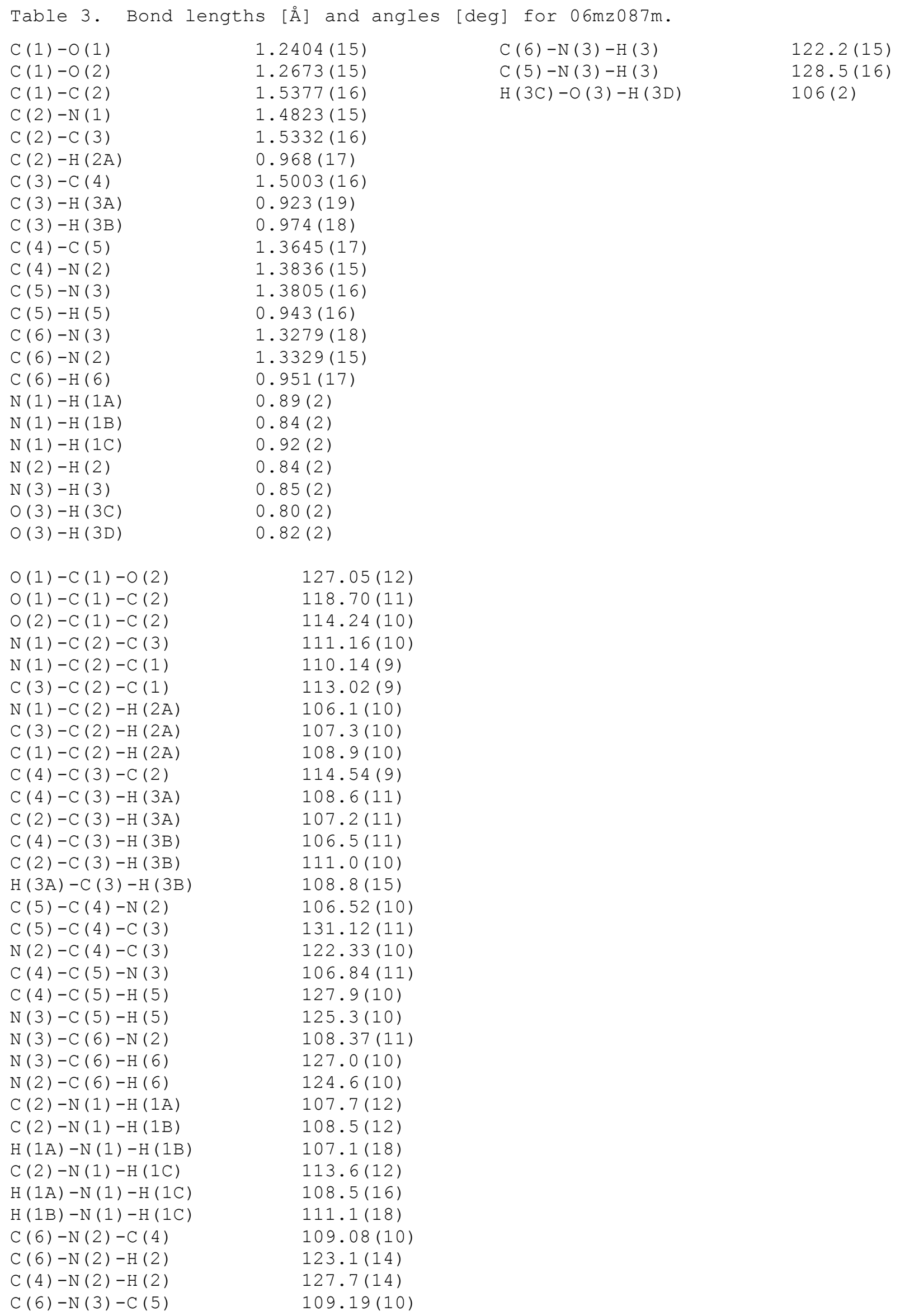


Table 4. Anisotropic displacement parameters $\left[\AA^{2} \times 10^{3}\right]$ for $06 \mathrm{mz} 087 \mathrm{~m}$. The anisotropic displacement factor exponent takes the form: $-2 \pi 2$ [ $h$ $\left.\left.a^{*}\right)^{2} \mathrm{U} 11+\ldots+2 \mathrm{~h} k \mathrm{a}^{*} \mathrm{~b} * \mathrm{U} 12\right]$

\begin{tabular}{|c|c|c|c|c|c|c|}
\hline & & U22 & U33 & U23 & U13 & U12 \\
\hline$C(1)$ & $10(1)$ & $8(1)$ & $16(1)$ & $0(1)$ & $1(1)$ & $2(1)$ \\
\hline$C(2)$ & $11(1)$ & $10(1)$ & $12(1)$ & $0(1)$ & $0(1)$ & $-1(1)$ \\
\hline$C(3)$ & $12(1)$ & $10(1)$ & $13(1)$ & $0(1)$ & $2(1)$ & $0(1)$ \\
\hline$C(4)$ & $12(1)$ & $11(1)$ & $14(1)$ & $-2(1)$ & $1(1)$ & $1(1)$ \\
\hline$C(5)$ & $15(1)$ & $10(1)$ & $15(1)$ & $-1(1)$ & $1(1)$ & $0(1)$ \\
\hline$C(6)$ & $16(1)$ & $13(1)$ & $17(1)$ & $-1(1)$ & $-2(1)$ & $3(1)$ \\
\hline $\mathrm{Cl}(1)$ & $12(1)$ & $11(1)$ & $18(1)$ & $-2(1)$ & $1(1)$ & $0(1)$ \\
\hline $\mathrm{N}(1)$ & $12(1)$ & $10(1)$ & $15(1)$ & $0(1)$ & $1(1)$ & $-2(1)$ \\
\hline$N(2)$ & $12(1)$ & $12(1)$ & $16(1)$ & $-2(1)$ & $1(1)$ & $0(1)$ \\
\hline $\mathrm{N}(3)$ & $19(1)$ & $10(1)$ & $16(1)$ & $1(1)$ & $1(1)$ & $1(1)$ \\
\hline$O(1)$ & $17(1)$ & $13(1)$ & $15(1)$ & $0(1)$ & $3(1)$ & $-2(1)$ \\
\hline$O(2)$ & $13(1)$ & $20(1)$ & $19(1)$ & $-4(1)$ & $0(1)$ & $-4(1)$ \\
\hline$O(3)$ & $21(1)$ & $31(1)$ & $18(1)$ & $-7(1)$ & $-2(1)$ & $11(1)$ \\
\hline
\end{tabular}

Table 5. Hydrogen coordinates $\left(\times 10^{4}\right)$ and isotropic displacement parameters $\left(\AA^{2} \times 10^{3}\right)$ for $06 \mathrm{mz} 087 \mathrm{~m}$.

\begin{tabular}{lrrrr}
\hline & \multicolumn{1}{c}{ x } & y & z & U (eq) \\
\hline H(2A) & $-1200(30)$ & $7131(19)$ & $8069(10)$ & $16(4)$ \\
H(3A) & $-90(30)$ & $4990(20)$ & $7475(12)$ & $20(4)$ \\
H(3B) & $1770(30)$ & $5780(20)$ & $7812(11)$ & $18(4)$ \\
H(5) & $-1340(20)$ & $2639(17)$ & $8802(10)$ & $10(3)$ \\
H (6) & $4290(30)$ & $1740(20)$ & $9271(11)$ & $16(4)$ \\
H(1A) & $-530(30)$ & $7840(20)$ & $9419(11)$ & $24(5)$ \\
H(1B) & $1210(30)$ & $7610(20)$ & $8968(12)$ & $28(5)$ \\
H(1C) & $690(30)$ & $6520(20)$ & $9636(14)$ & $33(5)$ \\
H(2) & $4050(30)$ & $4050(20)$ & $8548(13)$ & $30(5)$ \\
H(3) & $1050(30)$ & $940(30)$ & $9496(14)$ & $40(6)$ \\
H(3C) & $6580(30)$ & $3830(20)$ & $7159(14)$ & $32(5)$ \\
H(3D) & $5650(30)$ & $3780(20)$ & $6415(14)$ & $32(5)$ \\
\hline
\end{tabular}


Table 6. Hydrogen bonds for $06 \mathrm{mz} 087 \mathrm{~m}$ [ $\AA$ and deg].

\begin{tabular}{|c|c|c|c|c|}
\hline $\mathrm{D}-\mathrm{H} \ldots \mathrm{A}$ & $d(D-H)$ & $d(H \ldots A)$ & $d(D \ldots A)$ & $<(\mathrm{DHA})$ \\
\hline $\mathrm{O}(3)-\mathrm{H}(3 \mathrm{D}) \ldots \mathrm{Cl}(1)$ & $0.82(2)$ & $2.36(2)$ & $3.1808(11)$ & $176(2)$ \\
\hline$O(3)-H(3 C) \ldots O(2) \# 1$ & $0.80(2)$ & $1.94(2)$ & $2.7431(14)$ & $175(2)$ \\
\hline $\begin{array}{l}\mathrm{N}(3)-\mathrm{H}(3) \ldots \mathrm{Cl}(1) \# 2 \\
115.2(17)\end{array}$ & $0.85(2)$ & $2.88(2)$ & $3.3357(11)$ & \\
\hline $\mathrm{N}(3)-\mathrm{H}(3) \ldots \mathrm{O}(1) \# 3$ & $0.85(2)$ & $2.05(2)$ & $2.8078(14)$ & $147(2)$ \\
\hline$N(2)-H(2) \ldots O(2) \# 1$ & $0.84(2)$ & $1.81(2)$ & $2.6340(14)$ & $170(2)$ \\
\hline $\begin{array}{l}\mathrm{N}(1)-\mathrm{H}(1 \mathrm{C}) \ldots \mathrm{Cl}(1) \# 4 \\
170.7(18)\end{array}$ & $0.92(2)$ & $2.26(2)$ & $3.1781(11)$ & \\
\hline $\begin{array}{l}N(1)-H(1 B) \ldots O(3) \# 5 \\
171.7(18)\end{array}$ & $0.84(2)$ & $1.93(2)$ & $2.7651(14)$ & \\
\hline $\begin{array}{l}N(1)-H(1 A) \ldots C l(1) \# 6 \\
151.6(15)\end{array}$ & $0.89(2)$ & $2.37(2)$ & $3.1802(11)$ & \\
\hline
\end{tabular}

Symmetry transformations used to generate equivalent atoms:

$\# 1 x+1, y, z \quad \# 2-x, y-1 / 2,-z+3 / 2 \quad \# 3 x+1 / 2,-y+1 / 2,-z+2$

$\# 4-x+1 / 2,-y+1, z+1 / 2 \quad \# 5-x+1, y+1 / 2,-z+3 / 2 \quad \# 6-x, y+1 / 2,-z+3 / 2$ 